\title{
Comparison of synthetic turbulence approaches for blade element momentum theory prediction of tidal turbine performance and loads
}

\author{
Michael Togneri ${ }^{\mathrm{a},}{ }^{*}$, Grégory Pinon ${ }^{\mathrm{b}}$, Clément Carlier ${ }^{\mathrm{c}}$, Camille Choma Bex ${ }^{\mathrm{c}}$, Ian Masters ${ }^{\mathrm{a}}$ \\ a College of Engineering, Swansea University, Singleton Park, Swansea, SA2 8PP, UK \\ ${ }^{\mathrm{b}}$ Normandie Université, UNIHAVRE, CNRS, LOMC, 7600, Le Havre, France \\ ' IFREMER, 150 Quai Gambetta, 62200, Boulogne s/ Mer, France
}

\section{A R T I C L E I N F O}

\section{Article history:}

Received 12 December 2018

Received in revised form

8 May 2019

Accepted 26 May 2019

Available online 5 June 2019

\section{Keywords:}

BEMT

SEM

Sandia

Tidal turbines

Turbulence

Simulation

\begin{abstract}
A B S T R A C T
Turbulence is a crucial flow phenomenon for tidal energy converters (TECs), as it influences both the peak loads they experience and their fatigue life. To best mitigate its effects we must understand both turbulence itself and how it induces loads on TECs. To that end, this paper presents the results of blade element momentum theory (BEMT) simulations of flume-scale TEC models subjected to synthetic turbulent flows. Synthetic turbulence methods produce three-dimensional flowfields from limited data without solving the equations governing fluid motion. These flowfields are non-physical, but match key statistical properties of real turbulence and are much quicker and computationally cheaper to produce. This study employs two synthetic turbulence generation methods: the synthetic eddy method and the spectral Sandia method. The response of the TECs to the synthetic turbulence is predicted using a robust BEMT model, modified from the classical formulation of BEMT. We show that, for the cases investigated, TEC load variability is lower in stall operation than at higher tip speed ratios. The variability of turbine loads has a straightforward relationship to the turbulence intensity of the inflow. Spectral properties of the velocity field are not fully reflected in the spectra of TEC loads.
\end{abstract}

\section{Introduction}

Turbulence is a significant concern for tidal energy converters (TECs), due to its importance in determining the fluctuating loads on turbine blades [1] and ultimately the fatigue life of the device and its components [2]. Studies have measured a variety of different parameters for characterising turbulence at real sites [3-6], but there is no absolute consensus on exactly which characteristics are the most important for prediction of TEC loads. Nonetheless, it is standard to make use of some measure of turbulence strength (such as turbulent kinetic energy, $k$, or turbulence intensity, TI) and some measure of the spatial extent of turbulent fluctuations, typically as integral length scale [1]. Translating turbulence to turbine loads is also an area of ongoing research: there have been several experimental and computational investigations that have examined the influence of turbulence on TECs or scaleddown TEC models (see for instance Refs. [7-9]).

This paper will present results from blade element momentum

\footnotetext{
* Corresponding author.

E-mail address: M.Togneri@swansea.ac.uk (M. Togneri).
}

theory (BEMT) simulations of a flume-scale tidal turbine model. BEMT is a well-established method of simulating horizontal-axis turbines that extract energy from a flowing fluid [10]; its simplicity means that it is capable of simulating far more cases in the same amount of time than other numerical approaches that model flow physics more completely. The BEMT model used here is a robust code developed at Swansea University that incorporates a number of modifications to classical BEMT theory [11,12]. Most significantly, for the purpose of simulating TEC response to turbulent flows, it is capable of incorporating unsteady, non-uniform inflow conditions [13].

A modeller of turbulence can choose to deal with the fluid physics in greater or lesser detail [14]. The most sophisticated turbulence models have significant predictive power and correspondingly require a great deal of computational resource [15], but for this study it is important to select an approach that reflects the strengths of BEMT itself: its ability to quickly investigate a wide range of flow cases and operating conditions. This ability loses its value if it is necessary to perform a computationally-expensive calculation to generate turbulent inflow condition before the TEC's response to those conditions can be simulated. This motivates 
the use of synthetic turbulence methods, which use a restricted set of parameters to generate a three-dimensional turbulence velocity flowfield. These characteristic parameters are typically the velocity covariances or the spectra, both of which can be obtained with conventional instrumentation in the field with a high degree of confidence [16]. A flowfield generated this way does not, in general, satisfy the governing equations of fluid motions and is therefore not a physically accurate representation of turbulence, but it does replicate certain statistical parameters of turbulence.

This study compares two synthetic turbulence approaches. The first is the synthetic eddy method (SEM), which approximates turbulence as a collection of randomly-placed 'eddies'. This was originally developed to generate inflow conditions for large eddy simulations, and has been used in this context to simulate loads and wakes for both wind and tidal turbines [17,18]. We also synthesise turbulence with a spectral method, which we also refer to as the Sandia method after Sandia National Laboratories where it was first developed [19]; this method, or a similar one, is implemented in commercial codes such as Tidal Bladed and open-source codes like NREL's TurbSim [20]. Details of these schemes are given in sections 2.3 and 2.4 .

We begin with a brief description of the BEMT model used, and then introduce the specific lab-scale TEC that is simulated in this study and the conditions in which it is operated. This is followed by a brief discussion of the methods used to generate synthetic turbulence, and an assessment of how well they are able to replicate turbulent properties. We then go on to examine the results of using these synthetic turbulent flowfields in terms of power and thrust coefficients, as well as distributed loads across turbine blades.

\section{Background}

\subsection{Blade element momentum theory model}

Blade element momentum theory (BEMT) is a well-established model of conventional horizontal-axis rotors that was originally developed in the 1930s for aircraft propellers [21], but is still used today for both wind [22] and tidal [23] turbine applications. Its basic principle is to determine the fluid dynamic forces on a rotor through the necessary equality of its role as a source/sink of axial and swirl momentum with the lift and drag forces on the blade sections. The specific BEMT model employed in this study is a wellvalidated, robust code developed at Swansea $[11,12]$ that uses an iterative constrained nonlinear optimiser to minimise the difference between these two analyses of the rotor. This difference is quantified by the parameters called 'induction factors' which are a non-dimensional measure of the change in axial and swirl velocity induced by the presence of the rotor; the solver is considered to have converged on a solution when the change in induction factor from one iteration to the next is less than the tolerance of $1 \times 10^{-6}$. This BEMT model also incorporates a quasi-unsteady inflow model, allowing simulation of a turbine in unsteady, non-uniform flow conditions.

\subsection{Case study}

We compare our BEMT predictions to a lab-scale turbine that has been extensively investigated at the IFREMER combined wave and current flume [24]. The turbine is a three-bladed design of 0.7 m diameter; its chord and twist distributions are shown in Fig. 1. A NACA 63418 section was used across the entire radius.

The experimental study reported in Ref. [24] investigated a wide range of flow conditions, but in the current paper we restrict ourselves to two of these cases, which we call 'low turbulence' and 'high turbulence'. In both cases, the mean flow velocity is $1 \mathrm{~ms}^{-1}$, and the only mean flow property that changes is the turbulence intensity (TI), denoted $I_{\infty}$. This is the ratio of the root mean square of the fluctuation velocity components to the mean velocity magnitude, expressed as a percentage. If we write the Cartesian components of the velocity field as $u, v, w$, and use $u^{\prime}, v^{\prime}, w^{\prime}$ to denote their fluctuation components, then TI can be defined as:

$T I=100 \sqrt{\frac{\frac{1}{3}\left(\left\langle u^{\prime 2}\right\rangle+\left\langle v^{\prime 2}\right\rangle+\left\langle w^{\prime 2}\right\rangle\right)}{\langle u\rangle^{2}+\langle v\rangle^{2}+\langle w\rangle^{2}}}$,

where angle brackets are used to denote time-averaged values. The 'low turbulence' case has a turbulence intensity of $I_{\infty}=3 \%$; the 'high turbulence' case has $I_{\infty}=15 \%$. In addition to TI we also characterise turbulence through its anisotropy ratio - that is, the relative magnitude of turbulent fluctuations in each direction. This was not directly measured in the flume study, but based on the theoretical predictions of Nezu and Nakagawa [25], which were later corroborated by field measurements taken by Milne et al. [4]. We assume that this ratio takes the value $\sigma_{u}: \sigma_{v}: \sigma_{w}=$ $1: 0.75: 0.56$, where $\sigma_{u}$ represents the standard deviation of the $u$ component of velocity and is calculated as $\sigma_{u}=\left\langle u^{\prime 2}\right\rangle^{\frac{1}{2}}$. Flume data on the integral lengthscale is not available; to strike a balance between loading due to rotor-scale fluctuations and loading due to chord-scale fluctuations, integral lengthscale was set to $40 \%$ of the turbine radius.In order to verify that our BEMT model is able to accurately capture the mean turbine performance, we simulate the turbine in plug flow over a range of tip speed ratio (TSR) values to obtain the conventional performance curves of power and thrust coefficients, $C_{P}$ and $C_{T}$. Lift and drag data for the NACA $63418 \mathrm{sec}-$ tion were taken from Abbott and Doenhoff [26], corresponding to a Reynolds number of $6 \times 10^{6}$. Comparisons of these results with flume measurements are shown in Fig. 2 . We see that there is very good agreement in the power coefficient across the entire TSR range, but due to the additional measured drag from the support structure the model's prediction of thrust coefficient are consistently lower than the measured values. An approximate correction for this can be made by treating the support structure as a simple cylinder that is partially immersed in the turbine wake, calculating the resultant drag and subtracting this from the measured results. This correction substantially improves the agreement between predicted and measured results, particularly for the low turbulence case. To test the sensitivity of the results to the rotor discretisation, a second simulation was performed with almost twice as many radial elements, going from 22 to 43 evenly-spaced elements per blade. The consequent change in performance was approximately $1 \%$, which was sufficiently small to conclude that results obtained with the coarser discretisation would be satisfactory.

\subsection{Synthetic eddy method}

The synthetic eddy method was originally developed by Jarrin et al. as a means of generating inflow conditions for large eddy simulations (LES) of fluid flow [27], and continues to see use in this role [28]. Its basic approach is to fill a volume of space with randomly-positioned 'eddies' that induce a velocity in their own vicinity. Each 'eddy' is defined by a shape function (for the current study, a compactly-supported tent function), a size based on the specified integral lengthscale, and a strength. By calculating appropriate values for these properties based on measurements from real flows (e.g., turbulent kinetic energy, anisotropy ratios, integral lengthscales etc.), the flowfield induced by the eddies will have, statistically, the same Reynolds stress tensor as the real flowfield it is based on. A full description of the underlying mathematics can be found in Ref. [29]. It is important to bear in mind 


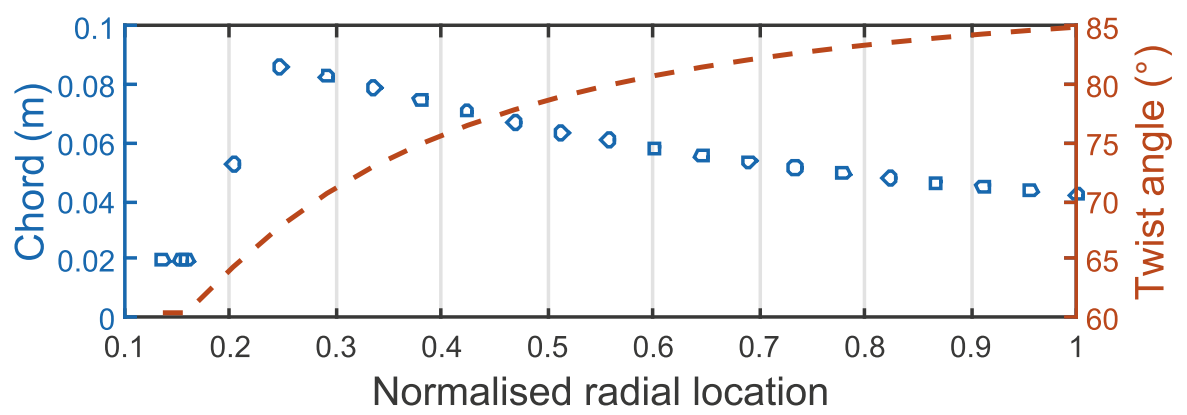

Fig. 1. Radial chord and twist distributions for the rotor used in the case study. Twist is defined as the angle between the chord line and the axis of rotation.
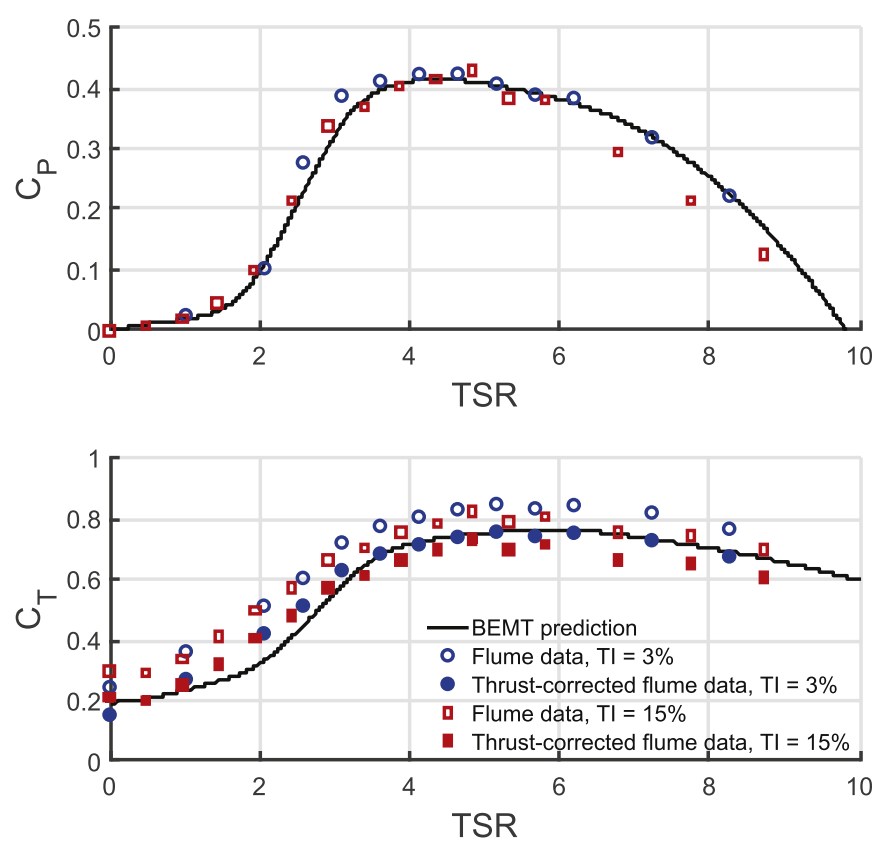

Fig. 2. Power and thrust coefficient dependence on TSR. Flume data are taken from Mycek et al. [24]. Filled symbols (for thrust only) indicate flume data corrected for the presence of the support structure, as described in the text. Experimental data points represent a time-average value over 100 s of measurement.

that, despite the name 'eddy', these velocity disturbances are not realistic representations of physical eddies and indeed are not intended to be; for clarity, we refer to them as pseudo-eddies.

\subsection{Sandia method}

The Sandia approach is a spectral method that was developed to simulate complete wind flowfields from a restricted set of measured data [19]; applying it to tidal stream flowfields is an intuitive extension of its original design purpose. The basis of the Sandia method is an array $\mathbf{S}$ whose every entry contains a power spectral density (PSD) corresponding to a pair of points. In this array, each diagonal entry corresponds to an auto-PSD, and each off-diagonal to a cross-PSD. We perform a Cholesky decomposition to define a matrix $\mathbf{H}: \mathbf{S}=\mathbf{H H}^{* T}$; then, by applying a random phase to each entry of $\mathbf{H}$, summing along the $n$th row will yield a series of Fourier coefficients corresponding to the nth point. These coefficients define a discrete spectrum which, when transformed back into the time domain, results in a time series of velocity with the correct spectral properties and spatial cross-correlations with all other points.
A key strength of the Sandia method is that it can work with any arbitrary spectrum, measured or theoretical. Observations have shown that turbulent flowfields in strong tidal currents tend to be well-described by the von Kármán spectrum [4], so this is employed here. In the absence of specific data on cross-spectra, Veers et al. [19] proposed a coherence function that relates the cross-PSD between any two points to their respective auto-PSDs and their separation distance, and we apply this approach here. Note that when generating a synthetic flowfield of finite duration, the spectrum is truncated compared to its theoretical value, resulting in a loss of energy. To mitigate this, we scale the finite time series according to the Rayleigh theorem to ensure that its power matches that of the full PSD. Lastly, an important shortcoming of the method is that each component of velocity is generated independently, which means that all cross-component correlations will be zero.

\section{Synthetic turbulence validation}

Before using the synthetic turbulent flowfields as inflow conditions to a BEMT model, we first verify that they are behaving as expected. We use flow conditions from the IFREMER flume tank experiments [24] as a benchmark for the synthetic turbulence methods. For all synthetic flowfields, the domain was defined as extending $10 \mathrm{~m}$ in the longitudinal direction, corresponding to $10 \mathrm{~s}$ of flow for a mean flow speed of $1 \mathrm{~ms}^{-1}$. The vertical and lateral extents of the synthetic flowfields were set to 1.5 times the diameter $D$ of the rotor (cf. the discussion of the turbine geometry in section 2.2). The grid spacing was $0.075 \mathrm{D}$ in the lateral and vertical directions, and a timestep of $0.01 \mathrm{~s}$ was chosen for the longitudinal direction, equivalent to a grid spacing of $0.0143 \mathrm{D}$. One hundred independent realisations of the turbulent flow were generated for both the high and low turbulence conditions with both synthetic turbulence methods. In Table 1 , we present the $I_{\infty}$ and anisotropy ratios for these benchmark flows. To obtain these data, we calculate a single mean value and standard deviation of both TI and

Table 1

Mean values and standard deviations of turbulence intensity $\left(I_{\infty}\right)$ and anisotropy ratio $\sigma_{u}: \sigma_{v}: \sigma_{w}$ for the SEM and Sandia methods compared to the benchmark turbulent flow cases.

\begin{tabular}{lll}
\hline & $I_{\infty}$ & $\sigma_{u}: \sigma_{v}: \sigma_{w}$ \\
\hline Target & $3 \%$ & $1: 0.75: 0.56$ \\
SEM & $2.97 \pm 0.03 \%$ & $1 \pm 0.01: 0.749_{ \pm} 0.008: 0.557_{ \pm} 0.008$ \\
Sandia & $3.00 \pm 0.05 \%$ & $1 \pm 0.01: 0.747 \pm 0.010: 0.557 \pm 0.005$ \\
Target & $15 \%$ & $1: 0.75: 0.56$ \\
SEM & $14.8 \pm 0.1 \%$ & $1 \pm 0.03: 0.750_{ \pm} 0.012: 0.558 \pm 0.013$ \\
Sandia & $15.1 \pm 0.2 \%$ & $1 \pm 0.02: 0.744_{ \pm} 0.013: 0.553 \pm 0.012$ \\
\hline
\end{tabular}


anisotropy ratio for each flowfield realisation, then tabulate the average of these across all realisations.

The synthetic turbulence methods are clearly able to satisfactorily replicate the key turbulence parameters of the benchmark turbulence cases. It is also instructive, however, to examine the spectral properties of the synthetic flowfields, and these are shown in Fig. 3. The Sandia method recovers the theoretical von Kármán spectrum far more accurately than SEM; this is unsurprising, as the Sandia method should replicate the spectrum used as a template. What is interesting is the manner in which SEM fails to accurately reproduce the expected PSD. Lower frequencies, corresponding to larger-scale motions, are relatively well-captured. However, as we move towards the frequencies comprising the inertial subrange, the PSD of the SEM turbulence exceeds the PSD of the von Kármán spectrum in magnitude for a narrow frequency range, approximately $1-4 \mathrm{~Hz}$. At frequencies above this range, the magnitude of the PSD drops sharply below that of the template spectrum, and from around $10 \mathrm{~Hz}$ upwards exhibits a series of linearly-spaced 'fringes'. These fringes closely approximate a $\operatorname{sinc}^{4}$ function, which is the PSD of a single tent function; the slight 'fuzziness' of the peaks in the SEM spectra is due to the fact that this PSD results from the superposition of several tent functions distributed randomly in three dimensions.

This non-physical behaviour of the SEM spectra at high frequencies can be ameliorated by varying the lengthscale of the pseudo-eddies [30]. The purple lines in Fig. 3 represent PSDs from SEM flowfields whose eddy lengthscales are governed by a normal distribution; the three different cases represent lengthscale distributions whose standard deviations are $25 \%, 50 \%$ and $75 \%$ of the mean value - lengthscales below zero are not permitted. Although modifying the SEM in this way means that the resultant flow does not strictly satisfy the mathematical conditions of the original formulation, the non-zero components of the Reynolds stress tensor of the resultant flowfields are still equal to those of the template data to within a standard deviation. Flowfields generated with modified SEM had a duration of 20s and a timestep of $0.0525 \mathrm{~s}$; this is the reason their spectra exist over a different frequency range to the Sandia and conventional SEM results.

\section{Results}

The effects of the low and high turbulence flows on the test turbine are examined for three different operating conditions: at power optimum (corresponding to a steady state characterised by
$\left.\mathrm{TSR}=4.4, C_{P}=0.4121, C_{T}=0.7418\right)$, in partial stall $(\mathrm{TSR}=2.5$, $C_{P}=0.2141, C_{T}=0.4326$ ) and in an overspeed condition (TSR $=8$, $\left.C_{P}=0.2316, C_{T}=0.7230\right)$. A simplified control scheme kept the rotor at a fixed TSR relative to the mean flow velocity. The results of these simulations are tabulated in Table 2 and presented graphically in Fig. 4. Analogous to the case in the validation results presented in section 3, the numbers shown here are calculated by taking the mean coefficients from simulations of the turbine response to each of the hundred independent realisations of each turbulent case, and calculating the mean and standard deviations of those populations. Note also that both thrust and power coefficients are normalised against the mean flow velocity for the whole simulation duration, rather than the instantaneous flow velocity at hub height.

These results illustrate several important phenomena. Firstly, none of the turbulent cases investigated resulted in a significant shift of the mean load characteristics. In every case, the mean load coefficients were within a single standard deviation of the corresponding steady state values. Secondly, the variability of the mean load coefficients appears to be related very straightforwardly to the turbulence intensity: moving from low turbulence to high turbulence (i.e., a fivefold increase from $I_{\infty}=3 \%$ to $I_{\infty}=15 \%$ ) also causes the standard deviation of the mean load coefficient to increase by a factor of approximately 5 - more precisely, the factor of proportionality varies in the range 4.6-5.8. This increase is roughly twice

Table 2

Mean value and standard deviations of power and thrust coefficients from BEMT simulations.

\begin{tabular}{lccc}
\hline Turbulence model & TI & \multicolumn{1}{c}{$C_{P}$} & \multicolumn{1}{c}{$C_{T}$} \\
\hline Stall region (TSR $=2.5$, steady $C_{P}=$ & 0.2140, steady $\left.C_{T}=0.4326\right)$ \\
SEM & $3 \%$ & $0.2114 \pm 0.0035$ & $0.4351 \pm 0.0057$ \\
& $15 \%$ & $0.1987 \pm 0.0188$ & $0.4359 \pm 0.0326$ \\
Sandia & $3 \%$ & $0.2117 \pm 0.0026$ & $0.4351 \pm 0.0055$ \\
& $15 \%$ & $0.2021 \pm 0.0145$ & $0.4397 \pm 0.0312$ \\
Optimal region (TSR $=4.4$, steady & $C_{P}$ & 0.4121, steady & $\left.C_{T}=0.7418\right)$ \\
SEM & $3 \%$ & $0.4129 \pm 0.0203$ & $0.7421 \pm 0.0208$ \\
& $15 \%$ & $0.4318 \pm 0.0942$ & $0.7403 \pm 0.0955$ \\
Sandia & $3 \%$ & $0.4175 \pm 0.0216$ & $0.7474 \pm 0.0225$ \\
& $15 \%$ & $0.4276 \pm 0.1027$ & $0.7425 \pm 0.1055$ \\
Overspeed region $\left(\mathrm{TSR}=8\right.$, steady $C_{P}=0.2316$, steady & $\left.C_{T}=0.7230\right)$ \\
SEM & $3 \%$ & $0.2326 \pm 0.0257$ & $0.7242 \pm 0.0274$ \\
& $15 \%$ & $0.2631 \pm 0.1341$ & $0.7428 \pm 0.1400$ \\
Sandia & $3 \%$ & $0.2324 \pm 0.0277$ & $0.7242 \pm 0.0297$ \\
& $15 \%$ & $0.2525 \pm 0.1400$ & $0.7375 \pm 0.1493$ \\
\hline
\end{tabular}

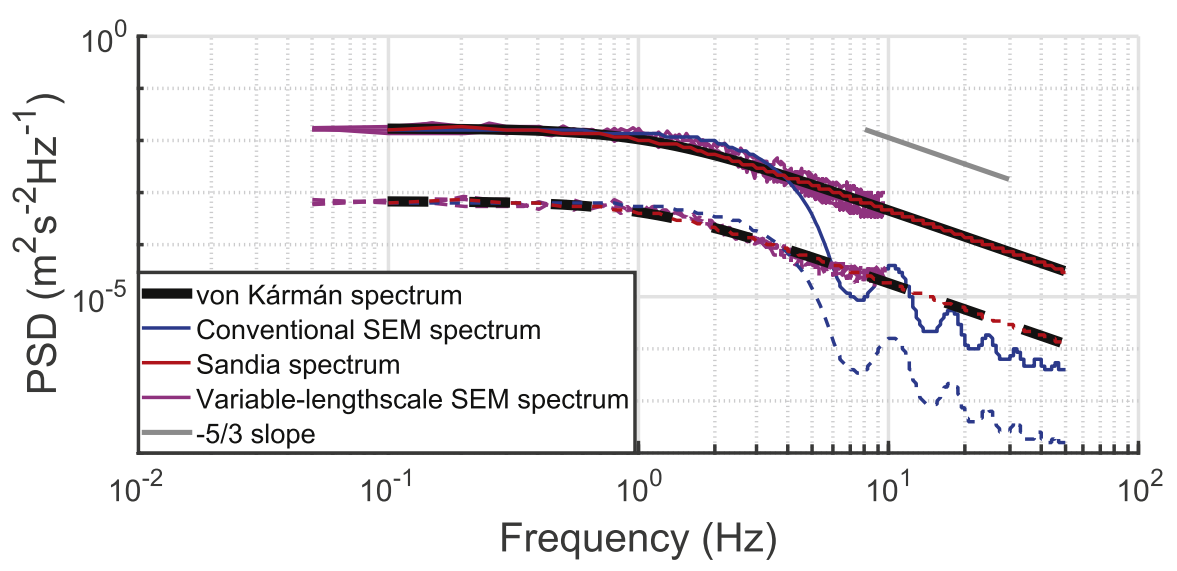

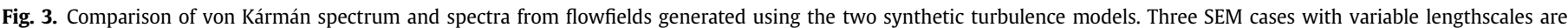

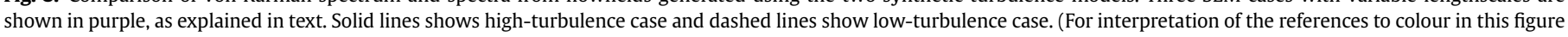
legend, the reader is referred to the Web version of this article.) 

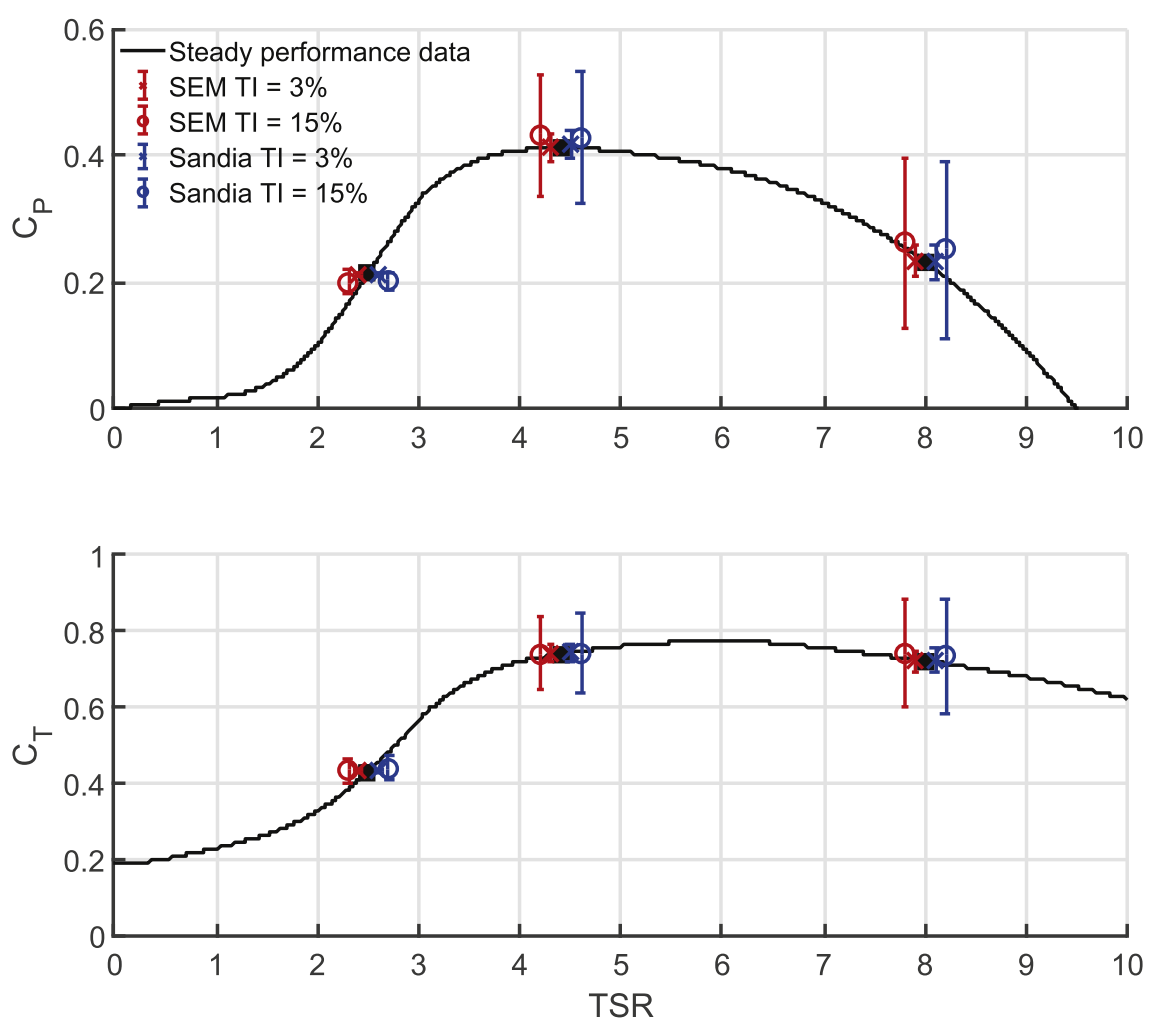

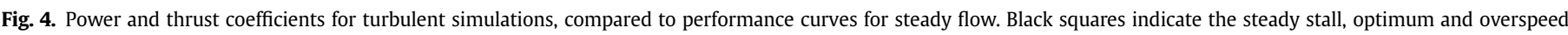
operating cases; coloured markers show mean coefficient values for turbulent cases with error bars indicating standard deviation. Turbulent data are offset in TSR for clarity.
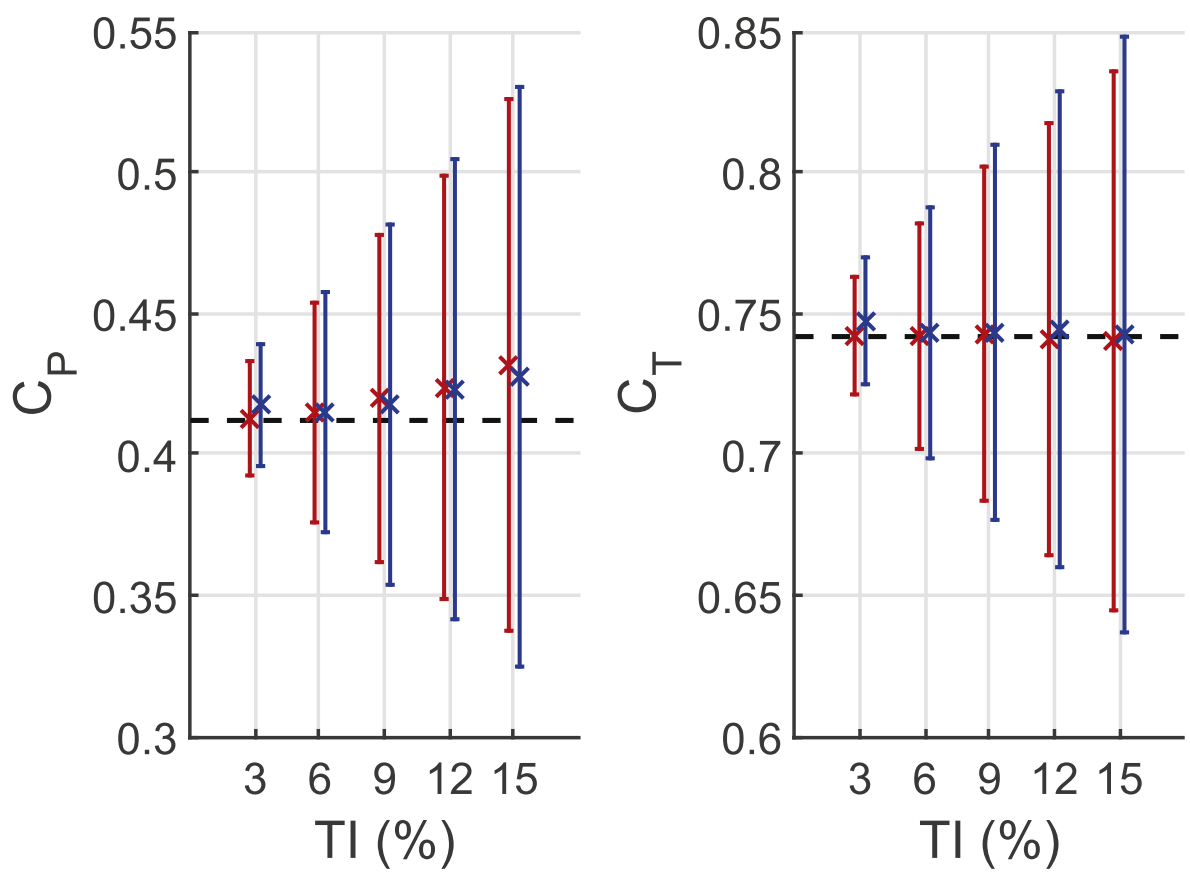

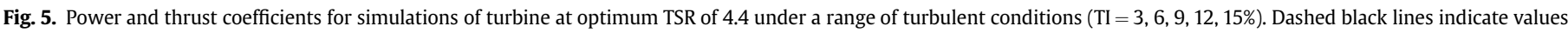

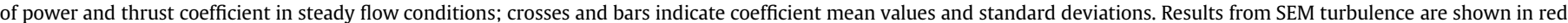

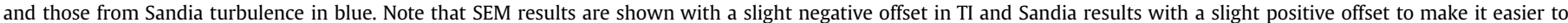

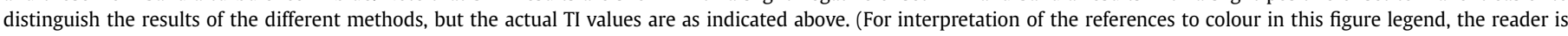
referred to the Web version of this article.) 
as large as that seen in the experiment of Mycek et al., whose results indicate that load coefficients increase by a factor of 2.7-2.9 between the low turbulence and high turbulence cases [24].

This proportionality is observed for intermediate values of the turbulence intensity as well, as can be seen in Fig. 5. This figure shows how the turbine power and thrust coefficients in optimum operation change for TI values between the $3 \%$ and $15 \%$ cases tested above. The mean values of the coefficients are not affected by the turbulence intensity - there is an apparent tendency for the mean $C_{P}$ value to increase with $\mathrm{TI}$, but the change is not statistically significant. The coefficient standard deviations, however, exhibit a clear linear dependence on turbulence intensity. Calculating a linear best fit between TI and standard deviation of the load coefficients shows that the constant of proportionality is 0.9 . Section 5 discusses this finding in greater detail.

It is also interesting to examine how variability of loads depends on operating conditions. The standard deviation of power and thrust coefficients does not differ greatly between optimum and overspeed operation, but drops significantly when the turbine is operated in the stall range. A similar discrepancy is also seen in the experimental results [24]. On the face of it, this is a surprising result: when the turbine is in the stall range (and therefore rotating more slowly), the blade-relative velocities will be more affected by the variations in inflow velocity. The same turbulent fluctuations would lead to greater variability in angle of attack; this is confirmed by examination of the angle of attack distributions shown in Fig. 6.

Nevertheless, this wider angle of attack range in stall operation does not lead to a wider range of hydrodynamic forces, as is visualised in more detail in Figs. 7 and 8, which show the average thrust and in-plane forces per unit length across all blades for all operating conditions and turbulence models. The standard deviations of these force distributions show the same tendency to be significantly greater in high-TSR operating conditions as was seen in the whole-turbine load coefficients. The results also make it very clear that the mean force distribution is almost unaffected by the choice of turbulence model. The effect on force variability is more significant - particularly in the high turbulence cases we see that the standard deviation of these force distributions is greater with SEM turbulence rather than Sandia turbulence.

In Fig. 9, we compare the spectral properties of the turbine power output and the inflow velocity. There are broad similarities in the shapes of the power and velocity spectra: in particular, we observe that the power spectra for Sandia turbulence cases tend to be greater in magnitude both at the high-frequency end and the extreme low-frequency end, while SEM turbulence yields a greater magnitude in the mid-range, just as is seen in the velocity spectra.

As was noted in section 3, modifying the SEM to permit variable lengthscales for the pseudo-eddies improves the spectral behaviour of the resulting synthetic turbulent flowfield. In the right-hand panels of Fig. 9, the average spectrum from all three of the variable lengthscale SEM cases investigated is plotted in purple alongside the spectra of the conventional synthetic turbulence models. The corresponding spectra of turbine power are plotted in the left-hand panels of the same figure. These spectra are noticeably noisier than those obtained with the Sandia or unmodified SEM turbulence models, but throughout most of the frequency range the modified SEM spectra are intermediate between the other two cases. Care should be taken in interpreting the behaviour of the power spectra for frequencies above $9.5 \mathrm{~Hz}$. This is the Nyquist frequency for the modified-lengthscale SEM velocity spectra, so the velocity spectrum is not explicitly specified by the input above this threshold.
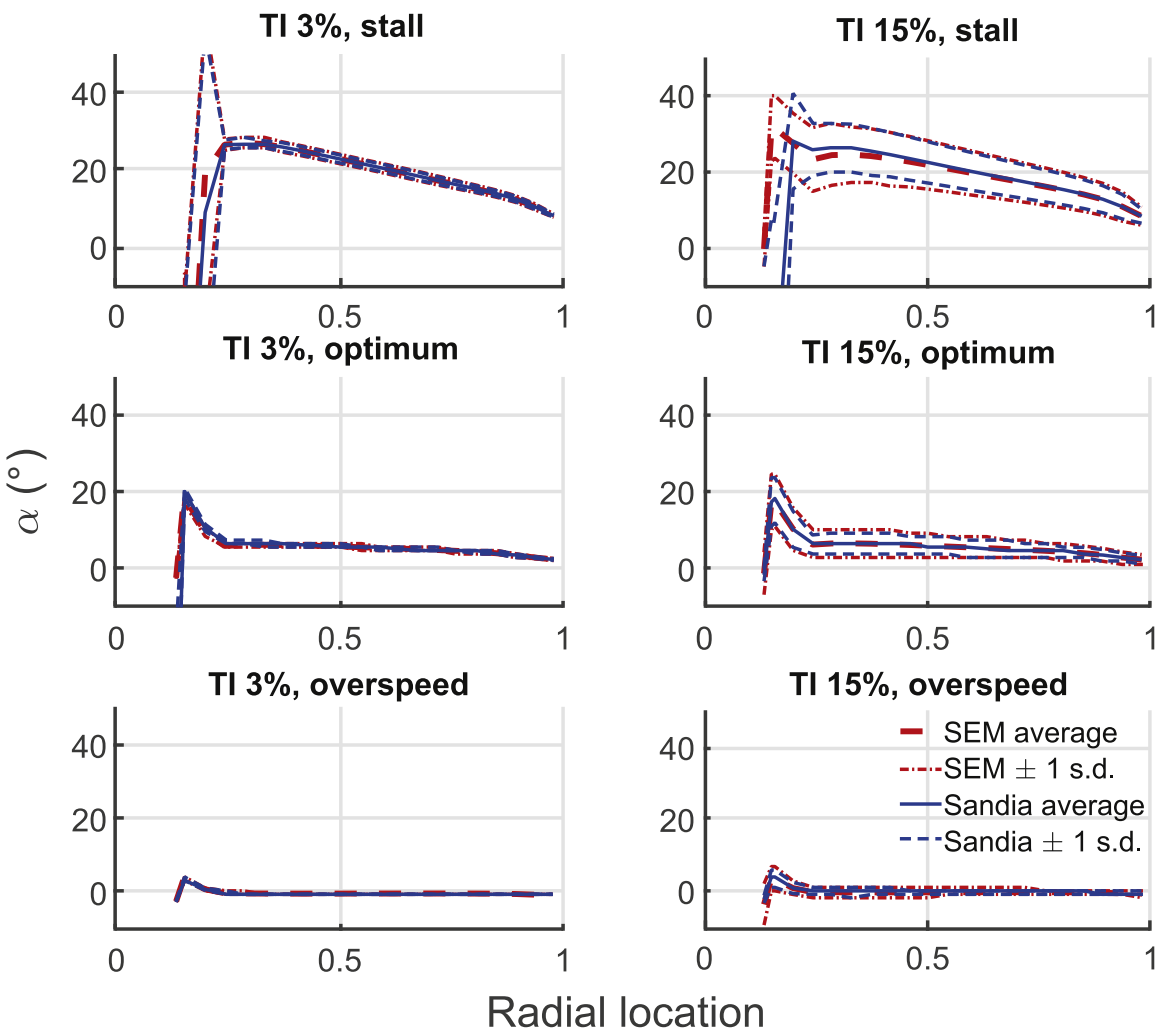

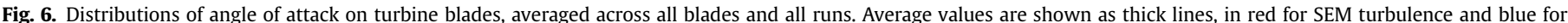

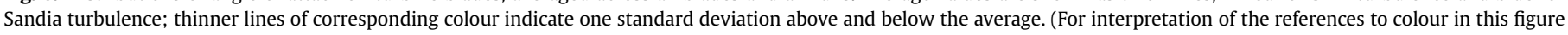
legend, the reader is referred to the Web version of this article.) 


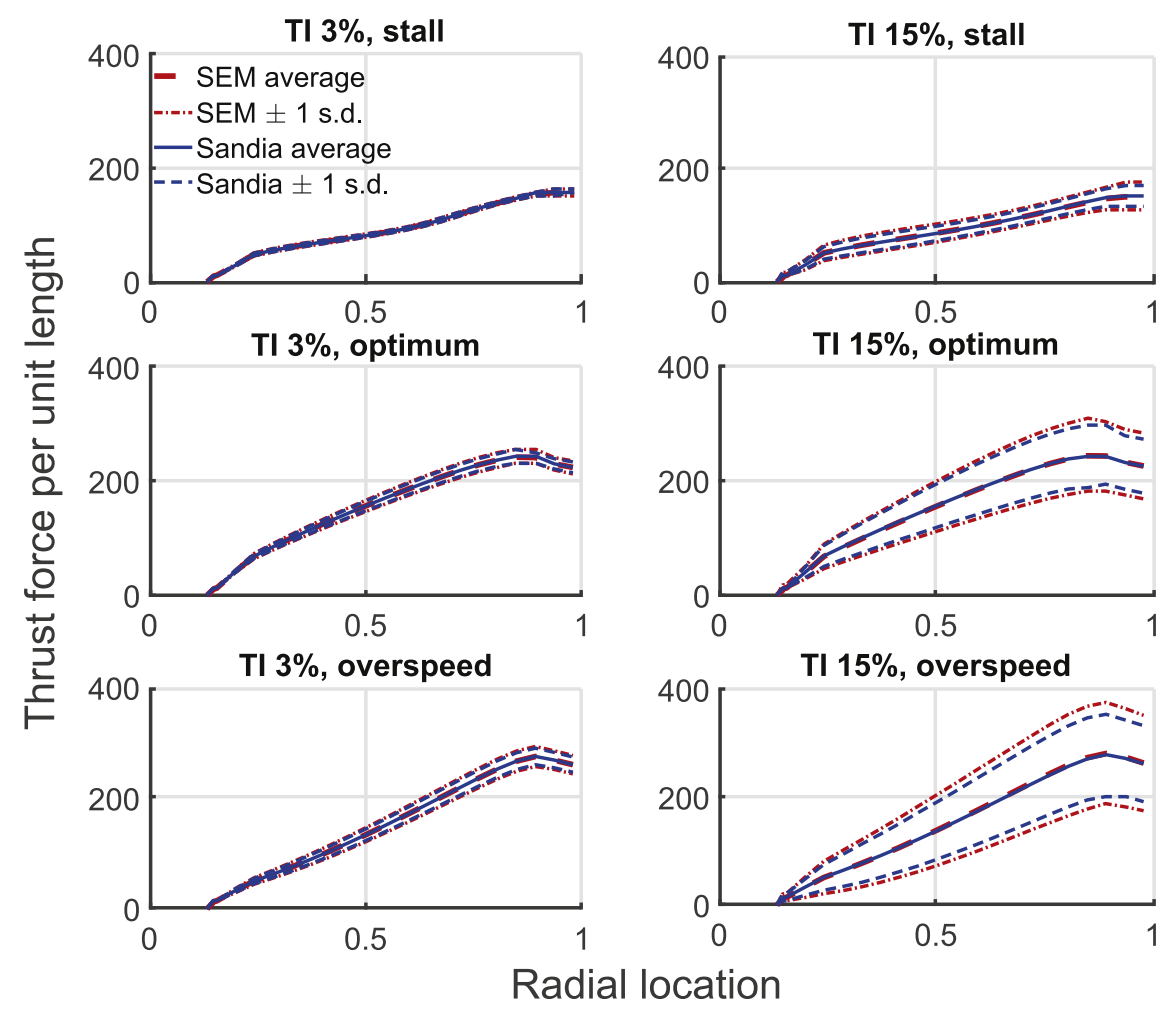

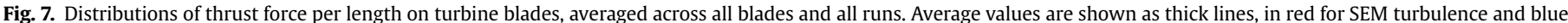

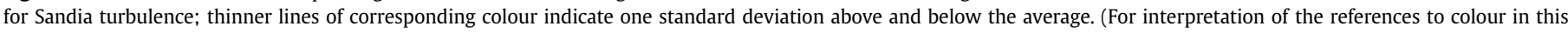
figure legend, the reader is referred to the Web version of this article.)

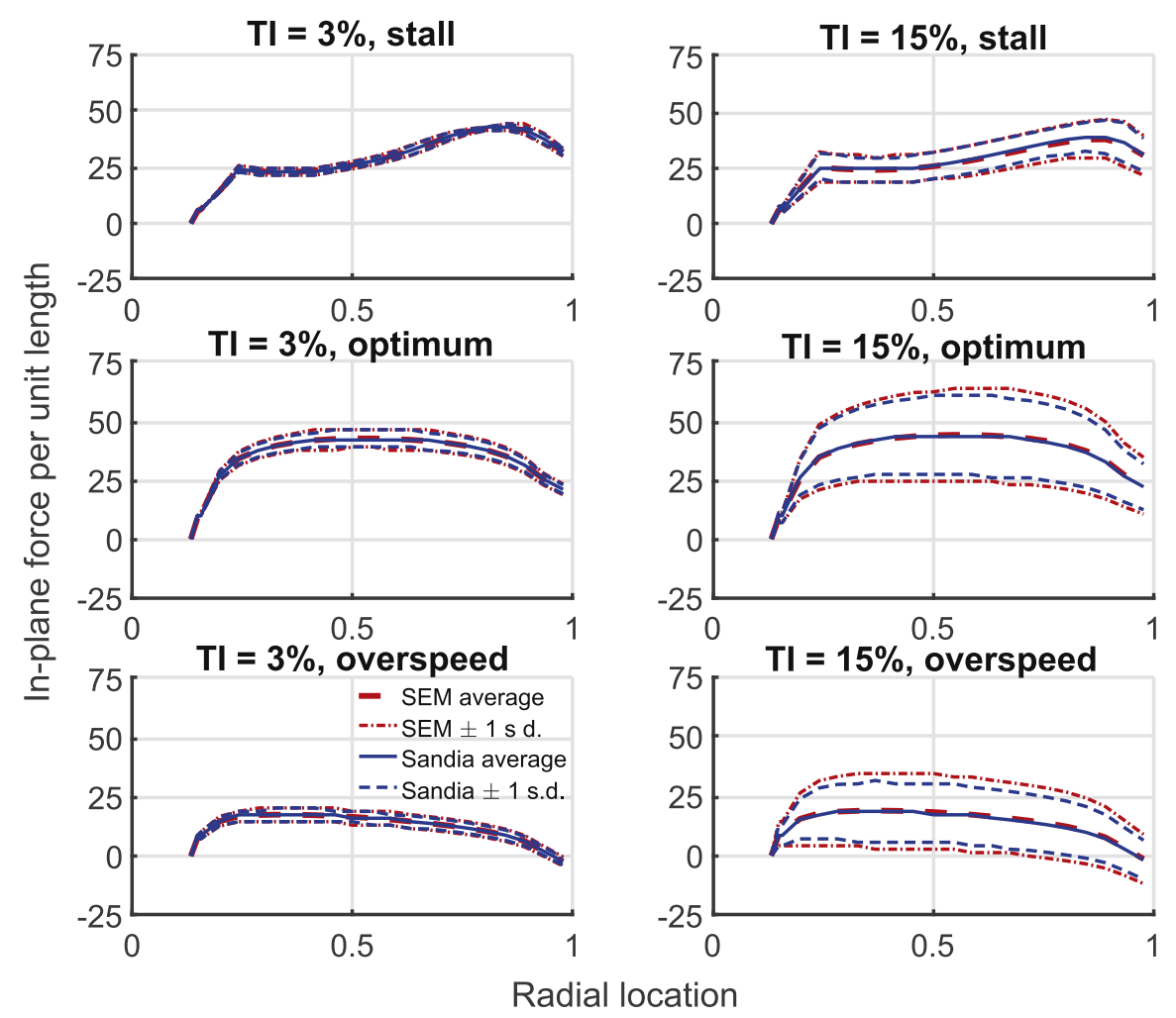

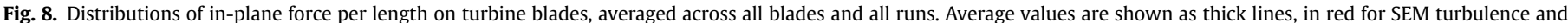

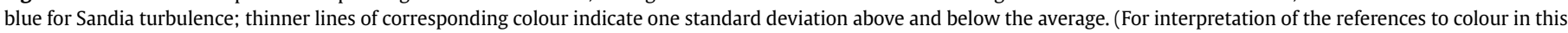
figure legend, the reader is referred to the Web version of this article.) 

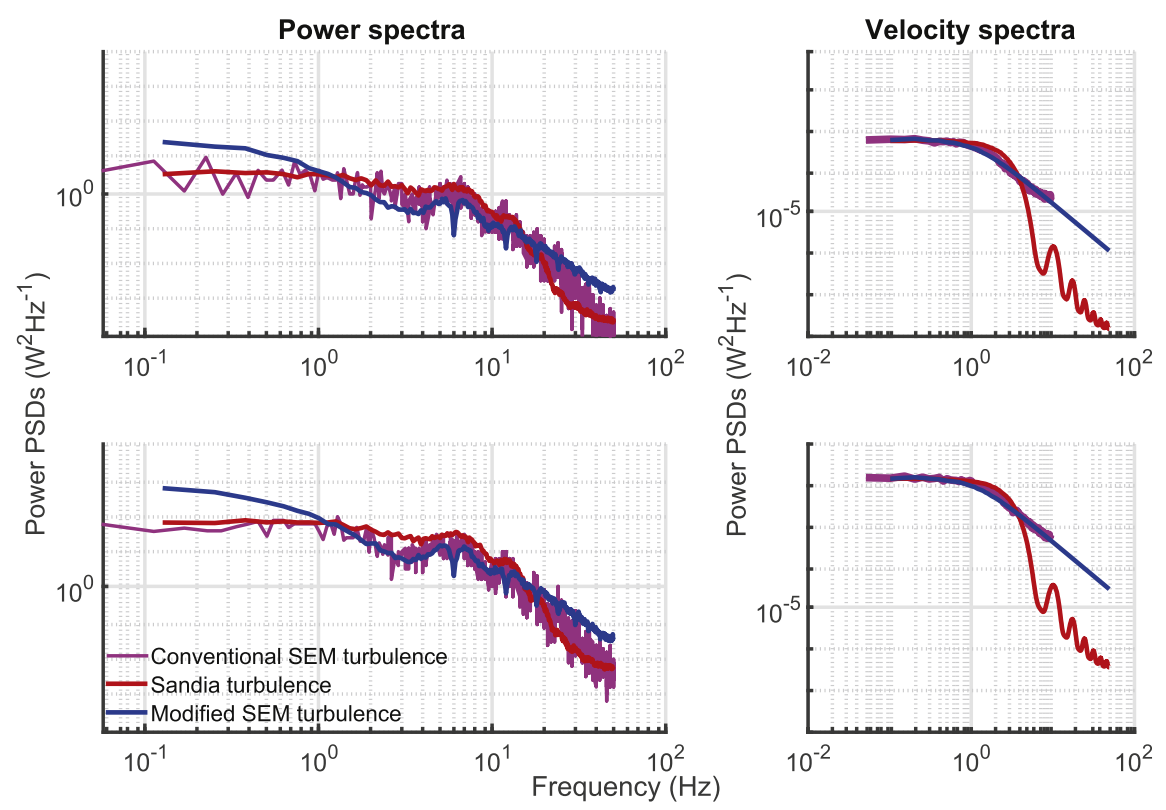

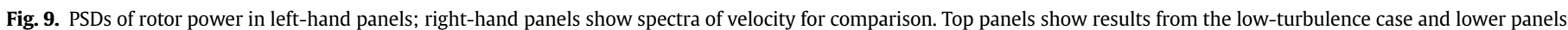
show results from the high-turbulence case.

\section{Discussion}

The clearest result from the simulations presented here is the very straightforward relationship between the turbulence intensity and the resultant standard deviation of the load coefficients. This was suggested by the results shown in Table 2 and Fig. 4 and reinforced by repeating the study for intermediate values of turbulence intensity. This increases our confidence in this observation, and allows us to state the relationship more precisely. The slope of a best fit line for normalised TI against normalised load coefficient standard deviations is between 0.908 and 0.893 , depending on which turbulence model and load coefficient is examined. More plainly, this implies that doubling the TI will increase the standard deviation of the load coefficients by a factor of 1.8. This is in excess of what is seen in the experimental results, which indicated that by doubling TI, the standard deviations would increase by a factor of around 1.45. Nonetheless, the corresponding Pearson correlation coefficients are all in excess of 0.99 , so we can be confident that a straightforward linear relationship is appropriate. This is a very promising result for predicting the effects of turbulence on turbine load.

To test the robustness of this result, we have repeated the same analysis for a second turbine design. The second turbine we have elected to study is one that has been scaled for a flume study of wake properties [31]. As a result, its power performance is quite different from what is expected in conventional design; nonetheless, as shown in Fig. 10, the BEMT code is able to satisfactorily predict its performance in steady flow over a range of TSR values. To test if the relationship between TI and turbine load variability holds for a different turbine, we have simulated this second turbine in optimum conditions (TSR $=4.45$, corresponding to a steady $C_{P}=$ 0.3278 and $C_{T}=0.8556$ ) at a range of TI values between $3 \%$ and $15 \%$, in the same manner as was done to produce the results of Fig. 5. The results of these simulations are visualised in Fig. 11.

The relationship observed is similar, although the slope of the best fit line is not the same as for the IFREMER turbine, and in this case the slope differs for power and thrust. The constant of proportionality between standard deviation of $C_{P}$ and TI is 0.686 if
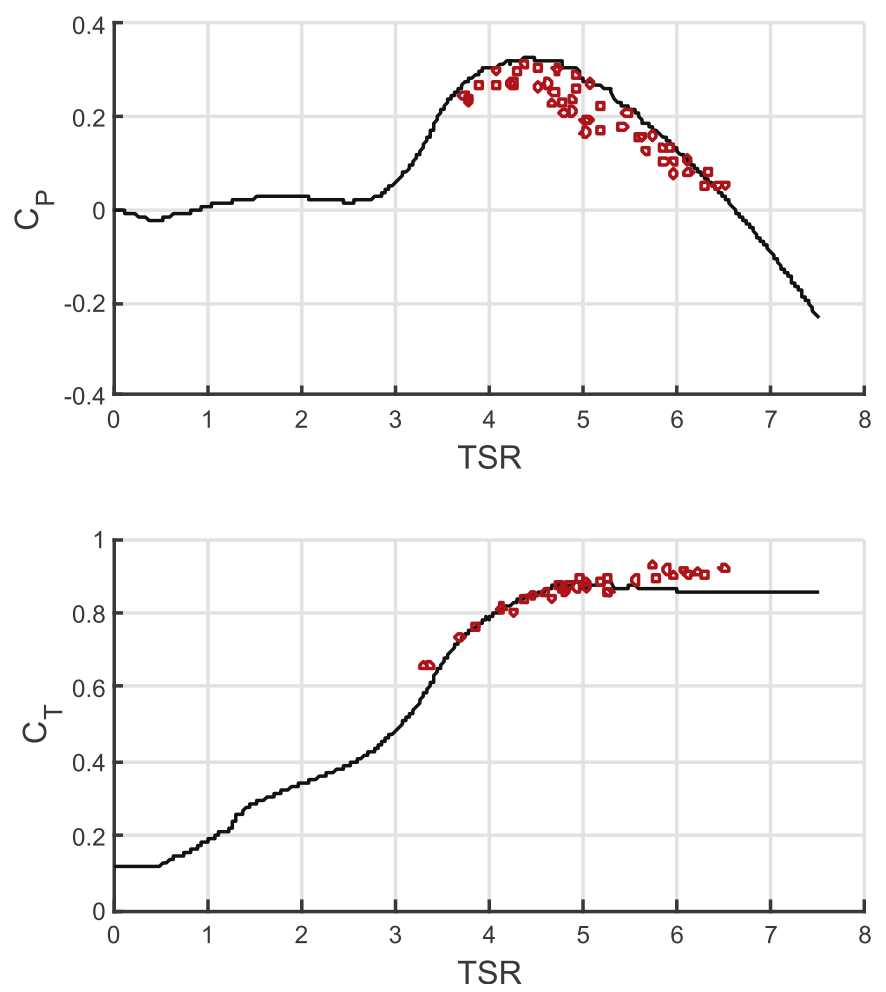

Fig. 10. Power and thrust coefficient dependence on TSR. BEMT predictions of load coefficients are shown as black lines, and flume measurements (taken from Stallard et al. [31]) are shown as hollow red circles. (For interpretation of the references to colour in this figure legend, the reader is referred to the Web version of this article.)

estimated from SEM turbulence, and 0.722 if estimated from Sandia turbulence; for $C_{T}$, the estimated constants of proportionality are 0.839 with SEM turbulence and 0.899 with Sandia turbulence. More importantly, however, they back up the observation that there is a straightforward linear relationship between turbulence 

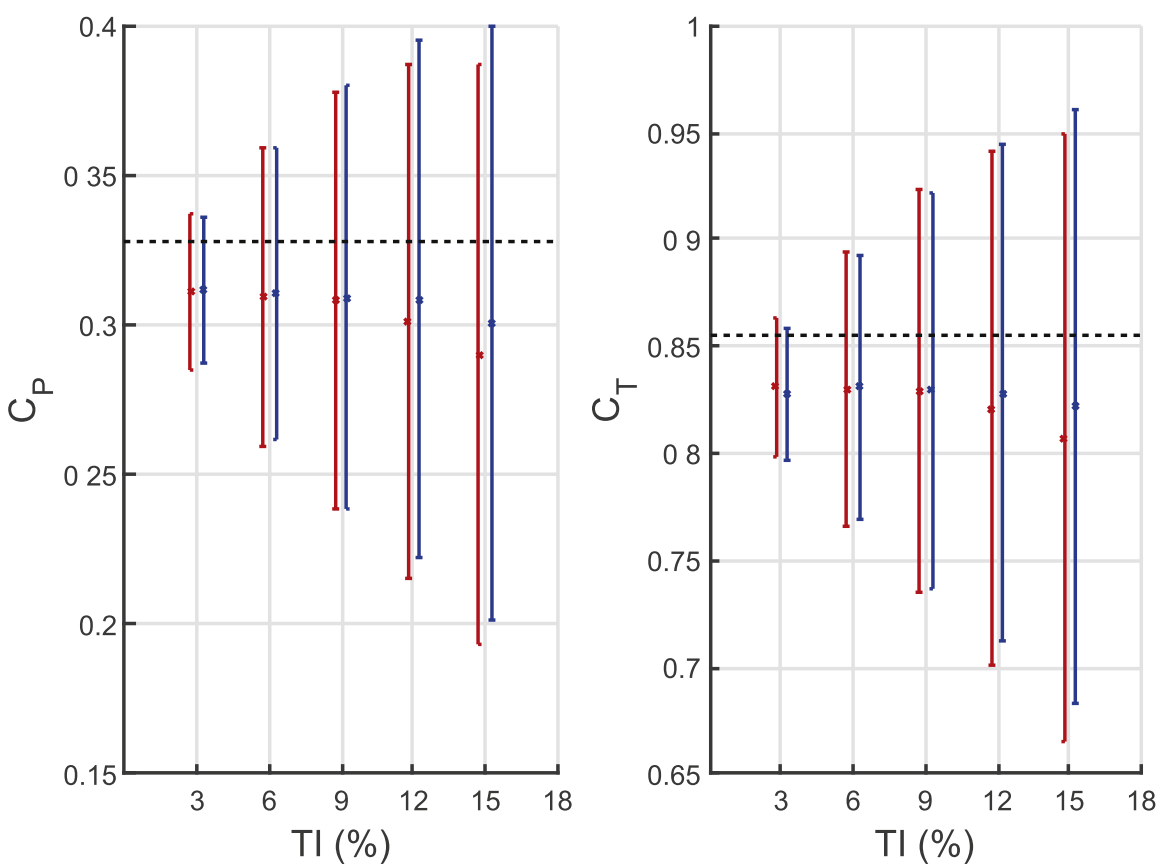

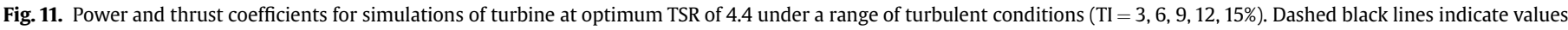

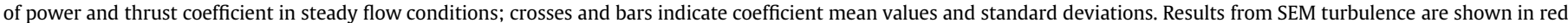

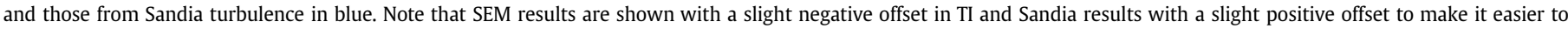

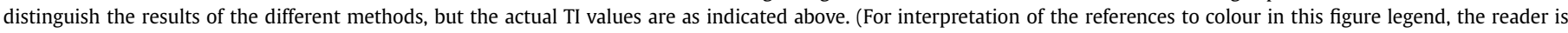
referred to the Web version of this article.)

intensity and the standard deviation of the load coefficients: the correlation coefficients for all datasets are again in excess of 0.99. This suggests that although the BEMT simulations have overestimated the increase in coefficient variability with TI for the IFREMER turbine compared to experimental data, we are correct to predict a linear relationship between these parameters.

More surprising is that, both here and in the experiments of Mycek et al. [24], load variability is less significant in stall operation than near optimum or in overspeed. This is unintuitive for two reasons: first, as mentioned above, in stall operation the rotational speed is low in comparison to the inflow velocity, so turbulent fluctuations will have a greater impact on the angle of attack of any given blade section. Secondly, in this operating condition more of the blade will be operating at angles of attack near stall. Changes in angle of attack are then more likely to mean that the blade will be rapidly switching between stalled and unstalled conditions, which would intuitively lead to greater variability in the overall hydrodynamic loads. Fig. 6 shows that angle of attack variability is indeed greater in stall operation; however, when we examine the variability of forces themselves (as in Figs. 7 and 8), the opposite tendency is seen. We have checked these results by recalculating the thrust and torque of a given blade element from the time series of angle of attack, in conjunction with the matching time series of the blade-relative velocity magnitude and the blade geometry. These recalculations confirm that the observed greater variability of angle of attack in stall operation does in fact correspond to a counterintuitive lower variability in the load coefficients.

A possible explanation for this could be found in the gentle stall characteristics of the NACA 63418 blade section used for the IFREMER rotor, as shown in Fig. 12. This shows that there is no large drop in lift after separation at approximately $14^{\circ}$. Thus, in stall operation, the variability of the forces resulting from the relatively wide angle of attack range is plausibly smaller than that in optimum or overspeed operation.

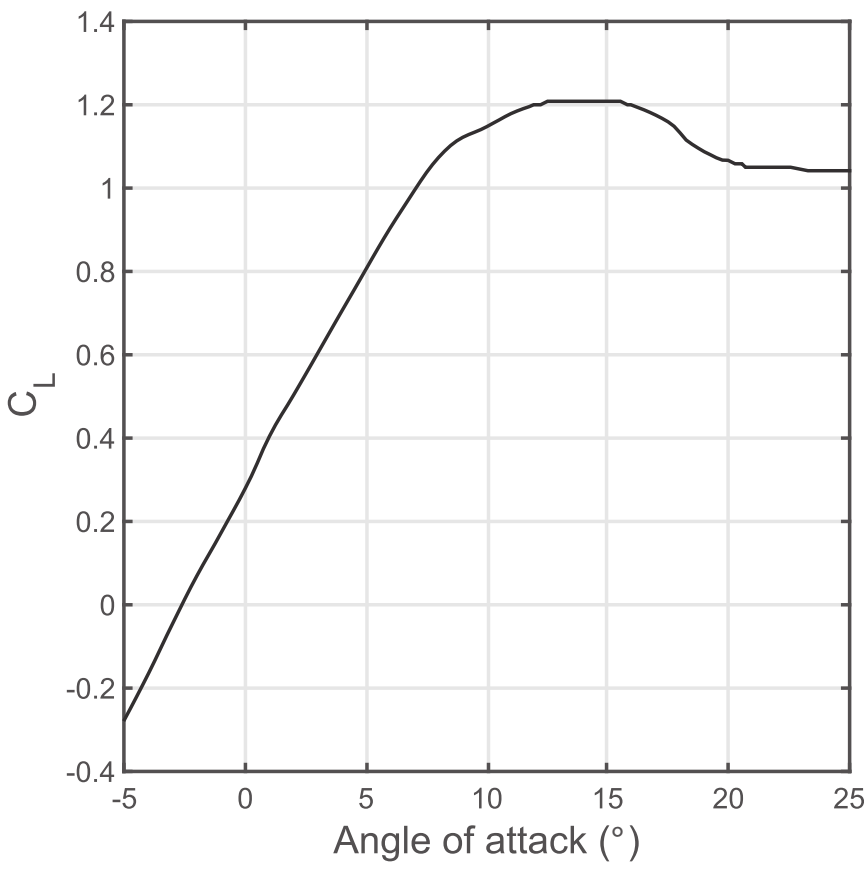

Fig. 12. Lift coefficient of the NACA 63418 blade section at $R e=6 \times 10^{6}$, plotted against angle of attack for a selected range of angle of attack values encompassing stall. Data taken from Ref. [26].

In Fig. 3, we compared the PSDs of the synthetic turbulent flowfields with one another and with the canonical von Kármán spectrum. Unsurprisingly, we found that the Sandia method does a very good job of replicating the template spectrum, while the SEM method does not. The variable-lengthscale modification to the SEM 
significantly improves the spectral properties of its flowfields. Averaging across multiple realisations (as seen in the right-hand panels of Fig. 9) smooths out the noisiness of the spectra from individual flowfields and shows a clear inertial subrange. Nonetheless, this improvement is not fully reflected in the spectra of the resulting turbine loads. A direct comparison is difficult, because fewer modified SEM cases were run and their spectra are correspondingly noisier; however, it appears that the varying the pseudo-eddy lengthscale does cause the spectrum of turbine power to shift towards its shape in the Sandia case, but by a much smaller margin than is seen for the velocity spectra.

This observation suggests that knowledge of the spectral properties of the turbulent flowfield is not sufficient to predict the spectral properties of the turbine loads for synthetic turbulence. This is similar to observations made of turbulent and turbine power spectra made in flume experiments [32], but there is an important distinction to be drawn. Experimental results indicate that as frequency increases, velocity and power fluctuations become less well correlated and so their spectra differ more significantly at higher frequencies. What this study shows is that, even where two different synthetic velocity fields have very similar spectral properties, the concomitant load spectra are not necessarily similar.

\section{Conclusions}

We have used two different synthetic turbulence methods to generate inflow conditions for a BEMT model of a lab-scale TEC. Both SEM and the Sandia spectral model of turbulence are shown to satisfactorily replicate certain statistical properties of real turbulent flow in the flume. Furthermore, although conventional SEM produces a flowfield whose velocity PSD diverges significantly from that of real flows, a simple modification to permit variablelengthscale pseudo-eddies is seen to address this shortcoming quite well. This modified SEM is not strictly guaranteed to replicate the template statistics as the classical formulation is, but we found that the Reynolds stress tensor does not significantly differ from the template values. The BEMT model has already been extensively validated [10-12], but the current study demonstrates that it captures the steady flow behaviour of the IFREMER test turbine to a high standard when compared to flume measurements.

Our simulations using the synthetic turbulence methods to define inflow conditions for the BEMT produced three key results. The most surprising result is that the variability of thrust and power for the turbine when operated at optimum TSR was reduced if the turbine is operated in stall. This runs counter to the observation that angle of attack variability is greater in stall operation, but we have confirmed that these two observations are consistent with one another. We hypothesise that this unusual result is caused by the gentle stall characteristics of the NACA 63418 section used in the IFREMER turbine.

Secondly, our simulations indicate that the variability of turbine loads, as measured by the standard deviation of the power and thrust coefficients, is directly proportional to TI. We calculated that this relationship has a constant of proportionality equal to 0.9 , and that its Pearson correlation coefficient is in excess of 0.99 , meaning that a linear relationship is strongly supported by the data. Simulations of a second turbine also supported a linear increase in load variability with TI with a high degree of certainty.

The third key result sheds some light on the spectral properties of TEC loads in simulations using synthetic turbulence. As mentioned above, the velocity PSD of SEM can be made more realistic with a variable lengthscale modification; however, the spectra of the turbine loads remain dissimilar. We conclude that, for simulations using synthetic turbulence, the spectral properties of the turbine loads cannot be predicted from knowledge of the corresponding velocity spectra alone.

\section{Acknowledgment}

The authors acknowledge the financial support of the Welsh Assembly Government and Higher Education Funding Council for Wales through the Sêr Cymru National Research Network for Low Carbon, Energy and Environment. The work was also supported by the EPSRC-funded projects "Extension of UKCMER Core Research, Industry and International Engagement" (EP/M014738/1) and "SURFTEC" (EP/P008682/1), and the ERDF-funded Interreg Atlantic Area project MONITOR (EAPA_333/2016). Clément Carlier would like to thank the Normandy Regional Council and IFREMER for the financial support of his Ph.D. grant. Camille Choma Bex acknowledges the financial support of IFREMER for her Ph.D. grant. The authors also would like to thank the CPER-ERDF program NEPTUNE also financed by the Normandy Regional Council. Finally the authors wish to thank the CRIANN (Centre Régional Informatiques et d'Applications Numérique de Normandie) for their available numerical computation resources.

\section{References}

[1] I. Milne, A. Day, R. Sharma, R. Flay, The characterisation of the hydrodynamic loads on tidal turbines due to turbulence, Renew. Sustain. Energy Rev. 56 (2016) 851-864.

[2] F. Elasha, D. Mba, M. Togneri, I. Masters, J.A. Teixeira, A hybrid prognostic methodology for tidal turbine gearboxes, Renew. Energy 114 (2017) 1051-1061.

[3] E. Osalusi, J. Side, R. Harris, Structure of turbulent flow in EMEC's tidal energy test site, Int. Commun. Heat Mass Transf. 36 (5) (2009) 422-431.

[4] I.A. Milne, R.N. Sharma, R.G. Flay, S. Bickerton, Characteristics of the turbulence in the flow at a tidal stream power site, Phil. Trans. Roy. Soc. Lond. A Math. Phys. Eng. Sci. 371 (1985) (2013) 20120196.

[5] J. Thomson, B. Polagye, V. Durgesh, M.C. Richmond, Measurements of turbulence at two tidal energy sites in Puget Sound, WA, IEEE J. Ocean. Eng. 37 (3) (2012) 363-374.

[6] M. Togneri, I. Masters, Micrositing variability and mean flow scaling for marine turbulence in Ramsey Sound, J. Ocean Eng. Mar. Energy 2 (1) (2016) $35-46$.

[7] T. Stallard, R. Collings, T. Feng, J. Whelan, Interactions between tidal turbine wakes: experimental study of a group of three-bladed rotors, Philos. Trans. R. Soc. A 371 (1985) (2013) 20120159.

[8] T. Blackmore, W. Batten, A. Bahaj, Influence of turbulence on the wake of a marine current turbine simulator, in: Proc. R. Soc. A, vol. 470, The Royal Society, 2014, p. 20140331.

[9] P. Pyakurel, J.H. VanZwieten, M. Dhanak, N. Xiros, Numerical modeling of turbulence and its effect on ocean current turbines, Int. J. Mar. Energy 17 (2017) 84-97.

[10] I. Masters, A. Williams, T.N. Croft, M. Togneri, M. Edmunds, E. Zangiabadi, I. Fairley, H. Karunarathna, A comparison of numerical modelling techniques for tidal stream turbine analysis, Energies 8 (8) (2015) 7833-7853.

[11] I. Masters, J. Chapman, J. Orme, M. Willis, A robust blade element momentum theory model for tidal stream turbines including tip and hub loss corrections, Proc. IMarEST-Part A-J. Mar. Eng. Technol. 10 (1) (2011) 25-35.

[12] J.C. Chapman, I. Masters, M. Togneri, J.A.C. Orme, The Buhl correction factor applied to high induction conditions for tidal stream turbines, Renew. Energy 60 (2013) 472-480.

[13] M. Togneri, I. Masters, G. Pinon, C. Carlier, C. Choma Bex, Comparison of synthetic turbulence approaches for two numerical tidal turbine models, in: 12th European Wave and Tidal Energy Conference, EWTEC 2017, 2017.

[14] S.B. Pope, Turbulent Flows, 2001.

[15] P. Moin, K. Mahesh, Direct numerical simulation: a tool in turbulence research, Annu. Rev. Fluid Mech. 30 (1) (1998) 539-578.

[16] M. Togneri, M. Lewis, S. Neill, I. Masters, Comparison of ADCP observations and 3D model simulations of turbulence at a tidal energy site, Renew. Energy (2017), https://doi.org/10.1016/j.renene.2017.03.061.

[17] I. Afgan, J. McNaughton, S. Rolfo, D. Apsley, T. Stallard, P. Stansby, Turbulent flow and loading on a tidal stream turbine by les and rans, Int. J. Heat Fluid Flow 43 (2013) 96-108.

[18] A. Creech, W.-G. Früh, A.E. Maguire, Simulations of an offshore wind farm using large-eddy simulation and a torque-controlled actuator disc model, Surv. Geophys. 36 (3) (2015) 427-481.

[19] P.S. Veers, Three-dimensional Wind Simulation, Tech. rep., Sandia National Labs, Albuquerque, NM (USA), 1988.

[20] J. Jonkman, L. Kilcher, TurbSim User's Guide: Version 1.06.00, Tech. rep., National Renewable Energy Laboratory, 2012.

[21] H. Glauert, Airplane propellers, in: Aerodynamic Theory, Springer, 1935, 
pp. $169-360$.

[22] S. Liu, I. Janajreh, Development and application of an improved blade element momentum method model on horizontal axis wind turbines, Int. J. Energy Environ. Eng. 3 (1) (2012) 30.

[23] W.M.J. Batten, A.S. Bahaj, A.F. Molland, J.R. Chaplin, The prediction of the hydrodynamic performance of marine current turbines, Renew. Energy 33 (5) (2008) 1085-1096.

[24] P. Mycek, B. Gaurier, G. Germain, G. Pinon, E. Rivoalen, Experimental study of the turbulence intensity effects on marine current turbines behaviour. part i: one single turbine, Renew. Energy 66 (2014) 729-746.

[25] I. Nezu, H. Nakagawa, Turbulence in Open-Channel Flows, Taylor \& Francis, 1993.

[26] I. Abbott, A. von Doenhoff, Theory of Wing Sections, Including a Summary of Airfoil Data, Courier Corporation, 1959.

[27] N. Jarrin, S. Benhamadouche, D. Laurence, R. Prosser, A synthetic-eddy- method for generating inflow conditions for large-eddy simulations, Int. J Heat Fluid Flow 27 (4) (2006) 585-593.

[28] K. Wilcox, J. Zhang, I. McLeod, A. Gerber, T. Jeans, J. McMillan, A. Hay, R. Karsten, J. Culina, Simulation of device-scale unsteady turbulent flow in the fundy tidal region, Ocean Eng. 145 (2017) 59-76.

[29] N. Jarrin, Synthetic Inflow Boundary Conditions for the Numerical Simulation of Turbulence, 2008.

[30] C. Carlier, Simulation du comportement d'hydroliennes : modélisation de l'influence de la turbulence ambiante et des effets d'interaction, 2017.

[31] T. Stallard, T. Feng, P. Stansby, Experimental study of the mean wake of a tidal stream rotor in a shallow turbulent flow, J. Fluids Struct. 54 (2015) 235-246.

[32] O.D. Medina, F.G. Schmitt, R. Calif, G. Germain, B. Gaurier, Turbulence analysis and multiscale correlations between synchronized flow velocity and marine turbine power production, Renew. Energy 112 (2017) 314-327. 